\title{
Article
}

\section{An online programme to reduce depression in patients with multiple sclerosis: a randomised controlled trial}

Fischer, Anja, Schröder, Johanna, Vettorazzi, Eik, Wolf, Oliver T, Pöttgen, Jana, Lau, Stephanie, Heesen, Christoph, Moritz, Steffen and Gold, Stefan M

Available at http://clok.uclan.ac.uk/16811/

Fischer, Anja ORCID: 0000-0002-7872-1292, Schröder, Johanna, Vettorazzi, Eik, Wolf, Oliver T, Pöttgen, Jana, Lau, Stephanie, Heesen, Christoph, Moritz, Steffen and Gold, Stefan M (2015) An online programme to reduce depression in patients with multiple sclerosis: a randomised controlled trial. The Lancet Psychiatry, 2 (3). pp. 217-223. ISSN 2215-0366

It is advisable to refer to the publisher's version if you intend to cite from the work. http://dx.doi.org/10.1016/S2215-0366(14)00049-2

For more information about UCLan's research in this area go to

http://www.uclan.ac.uk/researchgroups/ and search for <name of research Group>.

For information about Research generally at UCLan please go to http://www.uclan.ac.uk/research/

All outputs in CLoK are protected by Intellectual Property Rights law, including Copyright law. Copyright, IPR and Moral Rights for the works on this site are retained by the individual authors and/or other copyright owners. Terms and conditions for use of this material are defined in the policies page. 


\section{An online programme to reduce depression in patients with multiple sclerosis: a randomised controlled trial Anja Fischer*, Johanna}

Schröder*, Eik Vettorazzi, Oliver T Wolf, Jana Pöttgen, Stephanie Lau, Christoph Heesen, Ste en

Moritz†, Stefan M Gold†

Summary Background With a lifetime risk for major depressive disorder of up to $50 \%$, depression is a common comorbidity in multiple sclerosis but remains widely underdiagnosed and untreated. We investigated the potential of a fully automated, internet-based, cognitive behavioural therapy programme, Deprexis, to reduce depressive symptoms in patients with multiple sclerosis.

Methods For this randomised controlled trial, we recruited patients from an outpatient clinic in Hamburg, Germany. Patients aged 18-65 years were eligible for inclusion if they had multiple sclerosis and self-reported depressive symptoms. By use of a computergenerated randomisation sequence, we allocated 90 patients $(1: 1$; no blocking or strati cation) to either the intervention group or a waitlist control group for 9 weeks. The primary endpoint was the Beck Depression Inventory (BDI), as assessed by an intention-totreat analysis. This trial is registered with ClinicalTrials.gov, number NCT01663649.

Findings 71 patients completed the trial: 35 patients in the intervention group and 36 patients in the control group. During the intervention, BDI scores decreased in the Deprexis group and increased in the control group, yielding a positive e ect of Deprexis relative to the waitlist group (mean group di erence $-4 \cdot 02$ points [95\% CI $-7 \cdot 26$ to $-0 \cdot 79]$, $p=0 \cdot 015$, e ect size $d=0 \cdot 53$ ). Worsening of depressive symptoms from below to above the clinical cuto (BDI >13) occurred in three (7\%) of 45 patients in the control group and no patients in the Deprexis group. We noted no adverse events with respect to new occurrence of suicidal ideation during the trial.

Interpretation Psychological online-intervention programmes could be suitable for patients with multiple sclerosis who are unable to regularly attend therapeutic sessions because of mobility impairments.

Funding European Union and the Deutsche Forschungsgemeinschaft.

*These authors contributed equally and share rst authorship

$†$ Theseauthorscontributed equally and share senior authorship

Institute of Neuroimmunology and Multiple Sclerosis (INIMS), Center for Molecular Neurobiology (A Fischer PhD, 
C Heesen MD, S M Gold PhD), Department of Neurology (J Pöttgen MSc, S Lau MSc, C Heesen), Department of Psychiatry and Psychotherapy (J Schröder MSc, S Moritz PhD), Department of Medical Biometry and Epidemiology (E Vettorazzi MSc), Department of Medical Psychology

(S M Gold), University Hospital Hamburg-Eppendorf, Hamburg, Germany; and Department of Cognitive Psychology, Ruhr-University, Bochum, Germany (Prof O T Wolf PhD)

Correspondence to: Dr Stefan M Gold, Institute of Neuroimmunology and Multiple Sclerosis (INIMS), Centre for Molecular Neurobiology, University Medical Center Hamburg-Eppendorf, Falkenried 94, D-20251 Hamburg, Germany stefan.gold@zmnh. uni-hamburg.de

Multiple sclerosis is an in ammatory, demyelinating neurodegenerative disease of the CNS. In addition to motor dysfunction, visual and other sensory impairment, patients with the disorder often have neuropsychiatric symptoms such as depressed mood, fatigue, and cognitive impairment. Depression is especially common in this group of patients who have a lifetime risk for major de- pressive disorder as high as $25-50 \%,{ }^{1}$ and a 12 months' prevalence of up to $25 \%{ }^{2}$ Depression in multiple sclerosis has been linked to biological as well as psychological factors and substantially a ects psychosocial function. ${ }^{3}$ Depressive symptoms correlate with decreased quality of life, absence from work, and lower social support in patients with multiple sclerosis., ${ }^{4,5}$ Depression is also associated with lower immunotherapy adherence rates and might thus have direct consequences for overall health outcome. ${ }^{6}$ Moreover, depression is one of the main predict- ors for suicidal ideation and suicide risk in patients with muliple sclerosis. ${ }^{7}$ If left untreated, depressive symptoms in multiple sclerosis rarely remit spontaneously, often become chronic, ${ }^{8}$ and can worsen over time, particularly in patients with scores indicative of clinical depression at the study baseline. ${ }^{9}$ Despite its immediate clinical relevance, depression remains widely underdiagnosed and untreated in patients with multiple sclerosis. ${ }^{10}$

We are aware of only two previous placebo-controlled, randomised trials to have assessed the e ects of pharmacotherapy with desipramine and paroxetine in depression in patients with multiple sclerosis. ${ }^{11,12}$ Findings from a Cochrane review ${ }^{13}$ showed that some bene ts were apparent—albeit not statistically signi cant for most endpoints-but that adverse side- e ects such as nausea and headaches were a concern. Findings from a systematic review and meta-analysis suggested that cognitive behavioural therapy (CBT) might be bene cial in reducing depressive symptoms in patients with multiple sclerosis. ${ }^{14}$ However, the American Academy of Neurology's 2014 guidelines for psychiatric disorders in patients with multiple sclerosis have pointed out that more research is needed. ${ }^{15}$ In particular, a need exists to develop and rigidly test the e cacy of treatment strategies for multiple-sclerosis- associated depression and to facilitate access to these treatments. Because multiple sclerosis often causes motor impairment and decreased mobility as well as increased fatigue and cognitive problems, remote access options for psychotherapy might be useful to enhance availability of e ective depression inter- ventions such as CBT for these patients. For example, psychotherapy delivered by phone has been shown to decrease depressive symptoms in patients with multiple sclerosis. ${ }^{16,17}$ Such approaches, however, still require 
availability of a trained psychotherapist.

We tested the feasibility and e cacy of a fully automated, internet-based CBT (iCBT) programme (Deprexis) ${ }^{18}$ which has been shown to be e ective in patients with depression without comorbid somatic disorders, ${ }^{19}$ to reduce depressive symptoms in patients with multiple sclerosis.

\section{Methods}

\section{Study design and population}

In this randomised controlled trial, we contacted by mail patients registered in the database of the multiple sclerosis outpatient clinic at the University Medical Centre Hamburg-Eppendorf, Germany, whose scores from their last clinical visit indicated depressive symptoms (as measured by the mood subscale of the Hamburg Quality of Life Questionnaire for Multiple Sclerosis (HAQUAMS). ${ }^{20}$ Additionally, we recruited participants via online forums following guidelines of the Hamburg Department of Data Security. Participation was free of charge.

Patients were eligible for inclusion if they were aged 18-65 years, had a diagnosis of multiple sclerosis, self- reported depressive symptoms, and willingness to self- administrate iCBT sessions for 9 weeks. Participants were excluded if they did not consent to participation, were diagnosed with bipolar or schizophrenia spectrum disorders, had substantial neurocognitive impairment such as dementia, or had suicidal ideations. We used a secure online-survey programme EFS survey) to screen for inclusion and exclusion criteria before randomisation. A subgroup of the total sample volunteered to complete in-person visits to our multiple sclerosis outpatient centre before and after the intervention. The aim of this subgroup analysis was to validate depressive sympto- matology by means of additional questionnaires and structured interviews.

Written informed consent was provided via the online platform. The study was approved by the ethics committee of the German Society of Psychology.

\section{Randomisation and masking}

We allocated eligible patients to either intervention group or a waitlist control group (WLC; $1: 1$ ) using a computer- generated randomisation sequence with no strati cation or blocking. JS was in charge of generating the random sequence. None of the other investigators had access to the random sequence at any time. Patients were allocated

strictlyaccordingtotheorder(dateandexacttimeofday)

inwhichtheyhadcompletedtheonlinecheckofeligibility and baseline assessments of questionnaires as logged by the unipark system, ensuring that the eligibility check was blind to treatment allocation (concealed allocation). JS later was responsible for sending standardised email reminders to complete the online survey to every participant at pre-de ned times for follow-up assessment and contributed to writing the report. 
Masking of the participants regarding treatment allocation was not possible due to the nature of the information (ie, behavioural intervention). All outcome measures were collected via an automated online interface, so that masking of the assessors was not necessary. For the subgroup in which outcome measures were collected in person, we made substantial e ort to ensure that all patients were assessed by a clinician masked to group assignment—ie, the appointments were scheduled and patients were debriefed by sta members not involved in their assessment, and participants were instructed not to reveal their group assignment to the examiners at the clinic.

\section{Procedures}

Deprexis is an online programme based on principles of CBT. It consists of ten sequential modules_-psycho- education, behavioural activation, cognitive modi cation, mindfulness and acceptance, interpersonal skills, relaxation, physical exercise and lifestyle modi cation, problem solving, expressive writing and forgiveness, positive psychology, and emotion-focus interventions - plus an introduction and a summary module. It implements the technique of simulated dialogue by giving the user multiple-choice options and tailors the subsequent options to the patient's responses. The user's responses thus determine the course of each module. Dependent on the user's speed, each module can be completed in less than 60 mins. The programme is described in detail elsewhere. ${ }^{18}$ Participants randomly assigned to the control group were o ered full access to Deprexis on completion of the study.

\section{Outcomes}

All outcome measures - a set of questionnaires administered online to measure depressive symptoms, quality of life, and fatigue - were obtained at baseline

\section{Figure 1: Trial chart}

and 9 weeks after enrolment (ie, before and after the 9 week long programme for the Deprexis group and before and after waiting for 9 weeks for the waitlist group). We did a follow-up assessment 6 months after the end of the intervention using the same online platform.

Self-report questionnaires obtained online are similar to written questionnaires in terms of reliability and validity, ${ }^{21}$ as shown for depression scales including the Beck Depression Inventory (BDI). ${ }^{22}$

The primary endpoint of the study was severity of depressive symptoms as measured by the BDI. ${ }^{23}$ We analysed the BDI total score (primary endpoint) as well as BDI subscale scores (post-hoc analysis). The secondary endpoints were quality of life, as measured by the WHO Quality of Life scale (WHO-QoL BREF), ${ }^{24}$ disease-related quality of life (measured by HAQUAMS), ${ }^{20}$ and fatigue (Fatigue Scale For Motor And Cognitive Function; FSMC). ${ }^{25}$ We also screened for suicidal ideation and behaviour (with Suicide Behaviors Questionnaire- Revised [SBQ-R]), as a prede ned safety measure. ${ }^{26}$

In the clinical subgroup, patients underwent a structured clinical interview (The Mini International Neuropsychiatric Interview; MINI) ${ }^{27}$ administered by a trained clinician. They also 
completed an additional depression questionnaire (Inventory of Depressive Symptomatology; IDS-30SR). ${ }^{28}$ In this subgroup, we analysed the IDS total score as well as published IDS cognitive and somatic subscales. ${ }^{29}$

To assess treatment adherence we used data from the Deprexis interface, which tracks usage time of each participant. The usage log of Deprexis uses 5-min blocks and excludes each block of inactivity so that the logged usage times is a good estimate of time spent working with the programme. To assess the acceptance of Deprexis by patients, we obtained retrospective appraisal of the programme from participants randomised to the Deprexis group (appendix A).

We did not include a data monitoring committee to assess adverse events. However, we collected self- reported data for suicidal ideation and behaviour using the SBQ-R as a prede ned safety measure. If responses indicated acute risk of suicide (response 3a or 3b on SBQ-R item 3 plus score of 5 or 6 on SBQ-R item 4), patients were provided automatically with emergency contact numbers on the computer screen. To test for incidental evidence of adverse events, we also used a lower threshold for suicidal ideation (score $>1$ on item 9 of the BDI or score $>3$ on item 4 of the SBQ-R). We also regarded worsening of depressive symptoms during the trial from below to above the cuto for so-called caseness for clinical depression (BDI >13) as an adverse event.

\section{Statistical analysis}

Power calculation for the trial was based on results obtained from a previously published study using Deprexis in a cohort of patients with psychiatric disorders without comorbid somatic disorders. ${ }^{18}$ The pre-post

See Online for appendix A

-insert tabke here-

Data are n (\%) or mean (SD). HAQUAMS=Hamburg Quality of Life Questionnaire in Multiple Sclerosis. NaSSA=noradrenergic and speci c serotonergic antidepressant. SNRI=selective serotonin and noradrenaline reuptake inhibitors. SSRI=selective serotonin reuptake inhibitor. TCA=tricyclic antidepressants.

Table 1: Baseline characteristics

The effect size observed in that study was $d=0 \cdot 58$ and the between-group e ect size was $d=0 \cdot 64$. A power calculation with $80 \%$ power and $\alpha$ set at 0.05 with $d=0.64$ would result in a total sample size of 80 participants. We see Online for appendix B and C

using ANCOVA models. Here, change from baseline (calculated by subtracting pre-treatment scores from post-treatment scores) of the outcome measure was entered as the dependent variable and baseline scores as the covariate. As recommended by EMA, this model did not include treatment by covariate interactions. We treated missing data as last observation carried forward (LOCF) in the primary intention-to-treat analysis. We provide mixed models and multiple imputations (20 imputations using sex, age, group, and baseline BDI as predictors) as sensitivity analyses. We also include an analysis of patients with complete data before and after the treatment period (as per protocol). Raw data of the primary outcome BDI as well as imputed datasets are available in appendix B and appendix C, respectively. 
We analysed dichotomous endpoints indicating caseness (BDI below vs above the cuto ; diagnosis of major depressive disorder based on the MINI structured interview) using Cochran's $\chi 2$ test. All analyses of caseness were restricted to patients in whom caseness was established before and after intervention (as per- protocol sample). We used the categorical analyses to provide estimates for number needed to treat. Type I error was set at $5 \%$ and all tests were two-tailed. We provide e ect sizes as Cohen's d ([change Deprexis minus change WLC] divided by SD of the change score). We used PASW statistics software (version 18.0) for all analyses.

This trial is registered with ClinicalTrials.gov, number NCT01663649.

Figure 2: E ects of Deprexis on Beck Depression Inventory (BDI) scores Datapoints are mean score and error bars are SD.

conservatively estimated the sample size at 100 patients because the e ect size in psychiatric disorders might not readily be translated to patients with neurological disorders and comorbid depression.

Visual inspection of Q-Q plots suggested normal distribution of data. Therefore, we did statistical analyses using parametric tests. According to guidelines for statistical analysis of clinical trials published by the European Medicines Agency (EMA; codes CPMP/ ICH/363/96 and CPMP/EWP/2863/99), we did an intention-to-treat analysis for the primary outcome (BDI)

-insert table here-

Data are mean (SD) unless otherwise stated. BDI=Beck Depression Inventory. FSMC=Fatigue scale for Motor and Cognitive function. HAQUAMS=Hamburg Quality of Life Questionnaire for Multiple Sclerosis. WHO QoL BREF=WHO Quality of Life scale.

Table 2: Treatment effects

\section{Role of funding source}

The funding sources did not play any role in data collection, analysis, and interpretation of the data. The corresponding author had full access to all of the data and the nal responsibility to submit for publication.

\section{Results}

Patients were enrolled from July 16, 2012, to Oct 22, 2013. We screened 241 patients and randomised 90 to either the Deprexis or the control group ( gure 1). The number of patients who did not complete the 9 -week trial period was similar between the two groups (Deprexis $n=10$ [22\%]; control $n=9$ [20\%]). Baseline characteristics were much the same between the two groups (table 1).

Analysis of the primary endpoint BDI showed a statistically signi cant treatment e ect of Deprexis ( gure 2 and table 2). Group di erences were slightly larger in the mixed models, multiple imputations, and the per-protocol analyses (appendix A). 
BDI subscale analyses showed signi cant treatment e ects on the subdomains of negative attitude towards self and somatic symptoms (table 2).

We also analysed the BDI as a categorical variable (BDI 0-13 vs BDI >13) in the per-protocol sample post- hoc analysis). Between baseline and week 9, the number of patients above the cuto for clinical depression decreased in the Deprexis group and increased in the control group (p for between-group treatment di erence $=0 \cdot 01$; gure 3 ). Based on the BDI categorical analysis, the number needed to treat was eight.

34 participants (Deprexis $n=17$; control $n=17$ ) from the online cohort volunteered to undergo additional psycho- diagnostic assessment. In this subsample, we con rmed a positive treatment e ect of Deprexis on the BDI (LOCF $p=0 \cdot 047$; $d=0 \cdot 75$; appendix A). Similarly, depressive symptoms along DSM-IV diagnostic criteria as measured by the IDS showed a signi cant treatment e ect (LOCF $\mathrm{p}=0 \cdot 02 ; \mathrm{d}=0 \cdot 95$; appendix A). Furthermore, we detected treatment e ects in the IDS cognitive subscale ( $\mathrm{LOCF} p=0 \cdot 02, \mathrm{~d}=0 \cdot 88$ ) but not in the somatic subscale (appendix A). The proportion of patients meeting diagnostic criteria for major depressive disorder (as determined by the MINI, per protocol) decreased from baseline to 9 weeks but the di erence between Deprexis and control was not statistically signi cant ( $\mathrm{p}=0 \cdot 079$; appendix A). On the basis of the MINI analysis, the number needed to treat was three.

When assessing quality of life, as measured by the WHO-QoL BREF, we saw a treatment di erence only in the psychological wellbeing subscale (table 2). When assessing the Fatigue Scale For Motor and Cognitive Function (FSMC), we saw improvement with intervention versus control in only the motor fatigue subscale (table 2) but not on cognitive fatigue symptoms (table 2). We saw no di erence in any of the disease-speci c quality-of-life (HAQUAMS) subscales (table 2).

Figure 3: E ects of Deprexis on dichotomised Beck Depression Inventory (BDI) Patients were grouped as either below the cuto for clinical depression (BDI score $0-13$ ) or with scores indicative of clinical depression (BDI score $>13$ ).

On average, patients allocated to the Deprexis group spent 332 min (about $5 \cdot 5 \mathrm{~h}$ ) working with the programme (median 310 min; range 50-905). Patient satisfaction with the programme was high and most participants reported that the intervention met or exceeded their expectations (appendix A). Additionally, many of the participants indicated that they would recommend Deprexis to others with mild-to-moderate depression and speci cally those with multiple sclerosis. Only a few participants indicated that they needed assistance in handling the programme $(n=6,17 \%)$. However, about three-quarters $(n=27,77 \%)$ of the participants stated that they had to force themselves to complete all the sessions, and more than half $(n=18,51 \%)$ suggested that the programme might require some adjustments to suit the needs of patients with multiple sclerosis.

None of the enrolled participants met the prede ned criterion for acute risk of suicide (response 
3a or 3b on SBQ-R item 3 plus score of 5 or 6 on SBQ-R item 4) at baseline or the 9-week assessment. For the low-threshold de nition using BDI item 9, the criterion was not met by any patient in the Deprexis group (baseline $n=0 ; 9$ weeks $n=0$ ) but was met in one instance in the control group $(n=1 ; n=1)$. For SBQ-R item 4 , two patients met the low-threshold criterion at baseline and no patients met it at week 9 in the Deprexis group. In the control group, four patients met this criterion at both baseline and 9 weeks. However, using the low-threshold criteria, we saw no evidence of new occurrences of suicidal ideation during the trial in either group. We saw worsening of depressive symptoms during the trial from below to above the cuto for caseness (BDI >13) in three patients in the control group but none in the Deprexis group.

All 35 patients assigned to Deprexis and included in the 9-week analysis also completed the 6-months' follow-up examination. At 6 months, BDI scores in these participants were lower than at baseline (mean BDI at baseline 19·37 [SD 9.59]; mean BDI at 6 months 14.80 [10·03]; $\mathrm{p}=0 \cdot 001)$.

\section{Articles}

BDI score $0-13$

BDI score $>13100$

80604020

0

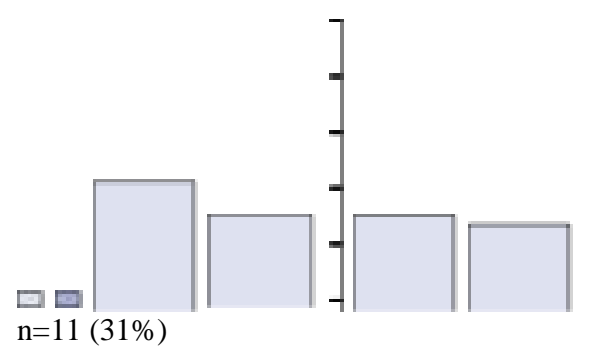

$n=16(46 \%)$

$n=12(33 \%)$

$n=11(31 \%)$
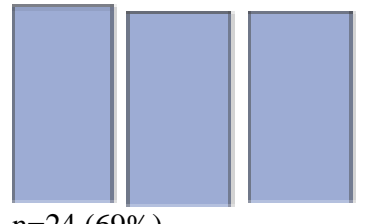

$n=24(69 \%)$

$\mathrm{n}=19(54 \%)$

$n=24(67 \%)$

$n=25(69 \%)$ 


\author{
Baseline Deprexis \\ 9 weeks \\ Baseline \\ 9 weeks \\ Control \\ Proportion of participants (\%)
}

\title{
Articles
}

\section{Panel: Research in context}

\section{Systematic review}

We searched Pubmed for relevant studies using the search terms "depress* AND multiple sclerosis AND (intern* OR comput*) AND (therap* OR intervention OR treatment)”. The search was restricted to English or German publications and carried out in April 2012, without date restrictions. We also browsed reference lists of relevant papers and our own libraries. We retrieved 80 articles, eight of which described trials of interventions in multiple sclerosis. Of these, two described internet-based interventions in multiple sclerosis (one uncontrolled study and one small randomised trial targeting fatigue in multiple sclerosis). Thus, we concluded that a phase 2 randomised controlled trial of an internet-based intervention for depression in multiple sclerosis was warranted.

\section{Interpretation}

Our findings show that a fully automated, internet-based cognitive behavioural therapy (CBT) intervention is feasible and effective in patients with multiple sclerosis and self- reported depressive symptoms, adding to evidence that CBT is an effective treatment strategy in multiplesclerosis-associated depression. The ability to provide patients with a self-guided, remote-access CBT intervention as shown in this trial provides new evidence that increases the range of therapeutic options available to such patients, at least as an interim solution until psychotherapy is available. Such options might be particularly relevant for patients with mobility or cognitive problems as well as pronounced fatigue, which could a ect their ability to regularly attend sessions with a therapist. Furthermore, it o ers patients who do not live close to care centres a low- barrier access to treatment. In times of increasingly restricted health-management costs, such online CBT interventions might o er a cost-e ective approach.

\section{Discussion}

We saw reduced depression scores, as measured by the BDI, in patients with multiple sclerosis participating in a 9-week iCBT intervention (Deprexis) compared with those not participating in 
the intervention. Importantly, Deprexis not only reduced severity of depressive symptoms, but also increased quality of life and decreased fatigue, adding to the clinical relevance of the ndings.

Our results are in accordance with the positive ndings reported in a recent, non-controlled pilot study on the feasibility of an online problem-solving programme in multiple sclerosis. ${ }^{30}$ Our results are also in line with randomised trials done in otherwise healthy patients with depression and without comorbid somatic disorders, which lends support to the usefulness of iCBT in general $^{31}$ and speci cally the Deprexis programme in patients with major depressive disorder. ${ }^{18,32,33}$

Few randomised trials have assessed the e ects of pharmacological or behavioural interventions to treat depression in patients with multiple sclerosis. Findings from studies using antidepressant pharmacotherapy in this population have suggested some e cacy but also indicatedfrequentside-e ects. ${ }^{13} \mathrm{CBT}$-basedpsychotherapy in group or individual settings is e ective in reducing depressive symptoms in multiple sclerosis. ${ }^{14}$ Moreover, CBT administered by telephone has shown promising results. ${ }^{16}$ Although this approach eliminates the need for the patient to travel, it still requires availability of a trained psychotherapist. In view of the motor impairment and

fatigability typically associated with multiple sclerosis, as well as the limited availability of psychotherapists, self- guided, automated, internet-based interventions such as Deprexis might be particularly useful for patients with multiple sclerosis and a need for depression treatment, at least as an interim solution until psychotherapy is available. Another obvious advantage of Deprexis and similar interventions is that they can be broken down into smaller modules and completed at any time, therefore allowing patients with increased fatigability or de cits in cognitive domains such as processing speed or attention to proceed at their own pace. Overall, retrospective, subjective ratings lend support to the feasibility and acceptance of Deprexis by patients with multiple sclerosis (appendix A).

Findings from a meta-analysis of iCBT interventions show that guided iCBT is more e ective than self-guided treatments and that even a small degree of contact with a clinician (eg, an interview or coming to the hospital for assessments) will lead to better outcomes. ${ }^{34}$ That the e ect size seen in the present study in a subgroup of patients undergoing psychodiagnostic assessment at our outpatient centre was larger than in the whole sample is in line with this possibility. Evidence has suggested that adding short face-to-face consultations in a blended-care design can have positive e ects on retention and outcome of iCBT. ${ }^{19}$ Email support might also be useful. ${ }^{33}$ Therefore, the e cacy of interventions such as Deprexis in multiple sclerosis might be further enhanced by adding therapist support in one form or another. Whether or not this is indeed bene cial remains to be tested in future studies and is an active area of investigation in our research.

Our study had some limitations. We selected parti- cipants on the basis of self-reported 
depressive symp- toms. A clinical diagnosis of major depressive disorder was not required for enrolment, which could have resulted in mild to moderate levels of depressive symptoms in participants. A previous study of Deprexis has reported strongest e ects in patients su ering from moderate depressive symptoms. ${ }^{32}$ Assessments of Deprexis in more severe depression in general and in multiple sclerosis speci cally are absent. Overall, the group di erences in our study were slightly smaller than the one reported before in patients with depression but no comorbid somatic disorder. ${ }^{18}$ This nding might be due to the lower level of depressive symptoms at baseline in our study. Alternatively, it could show the need to adjust Deprexis to multiple-sclerosis-speci c needs, as indicated by the retrospective appraisal of the programme in our patients. The drop-out rate in our study was just above the cuto for class-I evidence although within the range typically seen in behavioural interventions ${ }^{35}$ and low compared with other online interventions. ${ }^{36}$ Adherence to an online programme, though, is di cult to predict. ${ }^{37}$ Furthermore, the sample size was small, particularly in the clinical subgroup analyses. It remains to be seen if Deprexis e ects can be maintained when implemented at a larger scale outside of clinical trials.

This interventional study provides class-II evidence for the e cacy of an internet-based CBT intervention (Deprexis) for the treatment of depressive symptoms in patients with multiple sclerosis. ${ }^{38}$ This nding increases therapeutic options for depression associated with multiple sclerosis and might help to overcome treatment barriers such as mobility problems often encountered by patients with multiple sclerosis and other chronic health conditions (panel).

\section{Contributors}

AF, JS, CH, SM, and SMG designed the study. AF, JS, and SL obtained the data. AF, JS, EV, JP, OTW, CH, SM, and SMG did the analysis and interpretation of data. AF and SMG drafted the paper. JS, EV, OTW, JP, SL, CH, and SM revised the paper for intellectual content.

\section{Declaration of interests}

SMG reports personal fees from Novartis. All other authors declare no competing interests.

\section{Acknowledgments}

The research leading to these results has received funding from the European Union Seventh Framework Program (FP7/2007-2013) under grant agreement $n^{\circ}$ [268381]. SMG is supported by a Heisenberg Fellowship from the Deutsche Forschungsgemeinschaft (DFG, GO1357/4-1 and 5-1).

\section{References}

- 1 Siegert RJ, Abernethy DA. Depression in multiple sclerosis: a review. J Neurol Neurosurg Psychiatry 2005; 76: 469-75.

- 2 Patten SB, Beck CA, Williams JV, Barbui C, Metz LM. Major depression in multiple sclerosis: a population-based perspective. Neurology 2003; 61: 1524-27. 
- 3 Feinstein A, Magalhaes S, Richard JF, Audet B, Moore C. The link between multiple sclerosis and depression. Nat Rev Neurol 2014; 10: 507-17.

- 4 Hakim EA, Bakheit AM, Bryant TN, et al. The social impact of multiple sclerosis—a study of 305 patients and their relatives. Disabil Rehabil 2000; 22: 288-93.

• 5 José Sá M. Psychological aspects of multiple sclerosis. Clin Neurol Neurosurg 2008; 110: 868-77.

- 6 Tarrants M, Oleen-Burkey M, Castelli-Haley J, Lage MJ. The impact of comorbid depression on adherence to therapy for multiple sclerosis. Mult Scler Int 2011; 2011: 271321.

• 7 Feinstein A. Multiple sclerosis, depression, and suicide. BMJ 1997; 315: 691-92.

- 8 Arnett PA, Randolph JJ. Longitudinal course of depression symptoms in multiple sclerosis. J Neurol Neurosurg Psychiatry 2006; 77: 606-10.

- 9 Ensari I, Motl RW, McAuley E, Mullen SP, Feinstein A. Patterns and predictors of naturally occurring change in depressive symptoms over a 30-month period in multiple sclerosis. Mult Scler 2013; 20: 602-09.

- 10 Goldman Consensus G. The Goldman Consensus statement on depression in multiple sclerosis. Mult Scler 2005; 11: 32837.

- 11 Ehde DM, Kraft GH, Chwastiak L, et al. E cacy of paroxetine in treating major depressive disorder in persons with multiple $\quad$ sclerosis. Gen Hosp Psychiatry 2008; 30: 40-48.

- 12 Schi er RB, Wineman NM. Antidepressant pharmacotherapy of depression associated with multiple sclerosis. Am $J$ Psychiatry 1990; 147: 1493-97.

- 13 Koch MW, Glazenborg A, Uyttenboogaart M, Mostert J, De Keyser J. Pharmacologic treatment of depression in multiple sclerosis. Cochrane Database Syst Rev 2011; 2: CD007295.

- 14 Hind D, Cotter J, Thake A, et al. Cognitive behavioural therapy for the treatment of depression in people with multiple sclerosis: a $\quad$ systematic review and meta-analysis. BMC Psychiatry 2014; 14: 5.

- 15 Minden SL, Feinstein A, Kalb RC, et al. Evidence-based guideline: assessment and management of psychiatric disorders in individuals with MS: report of the Guideline Development Subcommittee of the American Academy of Neurology. Neurology 2014; 82: 174-81.

- 16 Mohr DC, Hart SL, Julian L, et al. Telephone-administered psychotherapy for depression. Arch Gen Psychiatry 2005; 62: 1007-14. 
17 Mohr DC, Likosky W, Bertagnolli A, et al. Telephone-administered cognitive-behavioral therapy for the treatment of depressive symptoms in multiple sclerosis. J Child Psychol Psychiatry 2000; 68: 356-61.

18 Meyer B, Berger T, Caspar F, Beevers CG, Andersson G, Weiss M. E ectiveness of a novel integrative online treatment for depression (Deprexis): randomized controlled trial. J Med Internet Res 2009; 11: e15.

19 Richards D, Richardson T. Computer-based psychological treatments for depression: a systematic review and meta-analysis. $J$ Clin Psychiatry 2012; 32: 329-42.

20 Gold SM, Heesen C, Schulz H, et al. Disease speci c quality of life instruments in multiple sclerosis: validation of the Hamburg Quality of Life Questionnaire in Multiple Sclerosis (HAQUAMS). Mult Scler 2001; 7: 119-30.

21 Ritter P, Lorig K, Laurent D, Matthews K. Internet versus mailed questionnaires: a randomized comparison. $J$ Med Internet Res 2004; 6: e29.

22 Hollandare F, Andersson G, Engstrom I. A comparison of psychometric properties between internet and paper versions of two depression instruments (BDI-II and MADRS-S) administered to clinic patients. J Med Internet Res 2010; 12 : e49.

23 Hautzinger M, Bailer M, Worall H, Keller F. Beck-Depressions- Inventar (BDI). Testhandbuch der deutschen. Ausgabe, Bern: Huber. 1995.

24 Skevington SM, Lotfy M, O’Connell KA, Group W. The World Health Organization’s WHOQOL-BREF quality of life assessment: psychometric properties and results of the international eld trial. A report from the WHOQOL group. Qual Life Res 2004; 13: 299-310.

25 Penner IK, Raselli C, Stocklin M, Opwis K, Kappos L, Calabrese P. The Fatigue Scale for Motor and Cognitive Functions (FSMC): validation of a new instrument to assess multiple sclerosis-related fatigue. Mult Scler 2009; 15: $1509-17$.

26 Osman A, Bagge CL, Gutierrez PM, Konick LC, Kopper BA, Barrios FX. The Suicidal Behaviors Questionnaire-Revised (SBQ-R): validation with clinical and nonclinical samples. Assessment 2001; 8: 443-54.

27 Ackenheil M, Stotz G, Dietz-Bauer R. Mini International Neuropsychiatric Interview. German Version 5.0.0, DSM-IV. München: Psychiatrische Universitätsklinik München, 1999.

28 Drieling T, Scharer LO, Langosch JM. The Inventory of Depressive Symptomatology: German translation and psychometric validation. Int J Methods Psychiatr Res 2007; 16: 230-36.

29 Duivis HE, Vogelzangs N, Kupper N, de Jonge P, Penninx BW. Di erential association of somatic and cognitive symptoms of depression and anxiety with in ammation: ndings from the Netherlands Study of Depression and Anxiety (NESDA). Psychoneuroendocrinology 2013; 38: 1573-85.

30 Boeschoten RE, Nieuwenhuis MM, van Oppen P, et al. Feasibility and outcome of a web-based self-help intervention for depressive symptoms in patients with multiple sclerosis: a pilot study. J Neurol Sci 2012; 315: 104-09.

31 Hedman E, Ljotsson B, Lindefors N. Cognitive behavior therapy via the Internet: a systematic review of applications, clinical e cacy and cost-e ectiveness. Expert Rev Pharmacoecon Outcomes Res 2012; 12: 745-64.

32 Moritz S, Schilling L, Hauschildt M, Schroder J, Treszl A. A randomized controlled trial of internet-based therapy in depression. Behav Res Ther 2012; 50: 513-21.

33 Berger T, Hammerli K, Gubser N, Andersson G, Caspar F. Internet-based treatment of depression: a randomized controlled trial comparing guided with unguided self-help. Cogn Behav Ther 2011; 40: 251-66.

34 Johansson R, Andersson G. Internet-based psychological treatments for depression. Expert Rev Neurother 2012; 12: 861-69.

35 Bados A, Balaguer G, Saldana C. The e cacy of cognitive-behavioral therapy and the problem of drop-out. J Clin Psychol 2007; 63: 585-92.

36 Andersson G, Stromgren T, Strom L, Lyttkens L. Randomized controlled trial of internet-based cognitive behavior therapy for 
distress associated with tinnitus. Psychosom Med 2002; 64: 810-16.

37 Kelders SM, Bohlmeijer ET, Van Gemert-Pijnen JE. Participants, usage, and use patterns of a web-based intervention for the prevention of depression within a randomized controlled trial. $\quad$ J Med Internet Res 2013; 15: e172.

38 Ashman EJ, Gronseth GS. Level of evidence reviews: three years of progress. Neurology 2012; 79: 13-14.

www.thelancet.com/psychiatry Published online February 4, 2015 http://dx.doi.org/10.1016/S2215-0366(14)00049-2 